\title{
Effect of the structure of polarimeter characteristic matrix on light polarization measurements
}

\author{
S.N. Savenkov ${ }^{1}$, Ye.A. Oberemok, O.S. Klimov, O.I. Barchuk \\ Taras Shevchenko Kyiv National University, Radiophysics Department, \\ 64, Volodymyrska str., 01601 Kyiv, Ukraine, \\ Phone: (380-44)526-05-80; e-mail: sns@univ.kiev.ua ${ }^{I}$
}

\begin{abstract}
In the paper, we carried out the comparative analysis of three polarimeters among the most usable their variants: (i) Stokes polarimeter based on phenomenological definition of Stokes parameters; (ii) Stokes polarimeter based on the method of four intensities; (iii) Stokes dynamic polarimeter. We show that, since the accuracy in determination of individual Stokes parameter is different for different types of polarimeters, and, therewith, it depends on polarization of input light. All that strongly motivates the choice of type of polarimeter to provide minimum errors in determination of polarization parameters (ellipticity angle $\varepsilon$, azimuth $\beta$, and degree of polarization $P$ ).
\end{abstract}

Keywords: Stokes vector, Stokes polarimeter, Stokes parameter.

Manuscript received 27.03.09; accepted for publication 14.05.09; published online 15.05.09.

\section{Introduction}

Polarization state of electromagnetic radiation changes when interacting with various media and is an additional source of information about their properties. Polarimetric methods through their high sensitivity (in particular to object anisotropy) could be used even at a negligible level of input light intensities. The time of measurement and errors are key factors in experimental investigation of different types of media by polarimetric methods [1-6]. The state of light polarization can be completely characterized by Stokes parameters, which allow describing both completely and partially polarized light. This makes the development of the systems measuring Stokes parameters (Stokes polarimeters) to be highly important for further improvement of polarimetric methods in medium investigations.

Up to date, it has been proposed a lot of schemes for Stokes polarimeter [7-10]. In all of them, to transform light polarization, used are polarization transformers with computer controlled parameters. The only polarimeter with division of intensities is exception [11], in which polarization transformers remain invariable. However, this results in considerable complication of the polarimeter calibration.

Stokes polarimeters with a mechanically controlled polarization transformer (e.g. with rotating optical elements) are useful in the view of realization simplicity, adjustment and exploitation. Now, the most usable variants of polarimeters are as follows: (i) Stokes polarimeter based on phenomenological definition of Stokes parameters [12]; (ii) Stokes polarimeter based on the method of four intensities [13]; (iii) dynamic Stokes polarimeter $[14,15]$. A plethora of papers $[10,13,16-$ $25]$ is devoted to polarimeter optimization in respect of gaining the minimal errors in measurements of Stokes parameters. In particular, derived were the optimal values of phase shifts and angular positions of phase plates. Also, it has been studied the question concerning the systematic error resulted from imperfectness of polarization elements used [22-24]. However, systematic analysis of random errors was not carried out for optimal regimes of the above types of Stokes-polarimeters. Besides, the most of papers, in which errors of polarimetric measurements are considered, supposes that the Stokes parameters are measured with an equal accuracy - but this is not the case in practice. In what follows, we show that individual errors in measurements of Stokes parameters depend on exact strategy of polarimetric measurements.

The aim of this paper is to estimate individual errors in measurements of Stokes parameters and effect of these errors on determination of light polarization parameters: ellipticity, azimuth and degree of polarization for the above mentioned strategies of polarimetric measurements. 


\section{Stokes polarimeter based on phenomenological definition of Stokes parameters}

According to phenomenological definition, four Stokes parameters $S_{1-4}$ have the following physical meaning: $S_{1}$ is a sum of light intensities that pass through polarizers with the orientation $0^{\circ}$ and $90^{\circ}$ (i.e. the total light intensity); $S_{2}$ - difference between the same intensities; $S_{3}$ - difference between intensities for light that pass through polarizers with the orientation $45^{\circ}$ and $135^{\circ} ; S_{4}$ - difference between intensities for light that pass through polarization elements transmitting light with either left or right circular polarization (for example, the quarter-wave plate and linear polarizer with the orientation $45^{\circ}$ or $135^{\circ}$ relative to fast axis of the plate). These definitions can be expressed in the form:

$S_{1}=I_{0^{\circ}}+I_{90^{\circ}} ; S_{2}=I_{0^{\circ}}-I_{90^{\circ}}$;

$S_{3}=I_{45^{\circ}}-I_{135^{\circ}} ; S_{4}=I_{L}-I_{R}$,

where $I_{\mathrm{x}}$ is the light intensity that pass through corresponding polarization elements.

The polarimeter operating accordingly to the definition Eq. (1) is presented in Fig. 1.

Here $\mathrm{P}$ is the polarizer with the azimuth $\theta$ and $\mathrm{PhPl}$ is the phase plate with the shift $\delta=\lambda / 4=90^{\circ}$ and azimuth $\alpha=0^{\circ}$, which can be introduced before the polarizer in the necessary steps of measurements.

The signal of photodetector (Fig. 1) depends on the position of polarization elements and the state of input polarization as follows:

$$
\mathbf{I}=\left[\begin{array}{c}
I_{0^{\circ}} \\
I_{90^{\circ}} \\
I_{45^{\circ}} \\
I_{135^{\circ}} \\
I_{L} \\
I_{R}
\end{array}\right]=\left[\begin{array}{c}
\left(\mathbf{M}_{P}\left(0^{\circ}\right) \cdot \mathbf{S}\right)_{1} \\
\left(\mathbf{M}_{P}\left(90^{\circ}\right) \cdot \mathbf{S}\right)_{1} \\
\left(\mathbf{M}_{P}\left(45^{\circ}\right) \cdot \mathbf{S}\right)_{1} \\
\left(\mathbf{M}_{P}\left(135^{\circ}\right) \cdot \mathbf{S}\right)_{1} \\
\left(\mathbf{M}_{P}\left(45^{\circ}\right) \cdot \mathbf{M}_{P h P l}\left(90^{\circ}, 0^{\circ}\right) \cdot \mathbf{S}\right)_{1} \\
\left(\mathbf{M}_{P}\left(135^{\circ}\right) \cdot \mathbf{M}_{P h P l}\left(90^{\circ}, 0^{\circ}\right) \cdot \mathbf{S}\right)_{1}
\end{array}\right],
$$

where $\mathbf{M}_{P}(\theta), \mathbf{M}_{P h P l}(\delta, \alpha), \mathbf{S}=\left[\begin{array}{llll}S_{1} & S_{2} & S_{3} & S_{4}\end{array}\right]^{T}$ denote the Mueller matrices of the polarizer as well as phase plate and Stokes vector for input light and have the form, correspondingly:

$$
\mathbf{M}_{P}(\theta)=\frac{1}{2}\left[\begin{array}{cccc}
1 & \cos (2 \theta) & \sin (2 \theta) & 0 \\
\cos (2 \theta) & \cos ^{2}(2 \theta) & \cos (2 \theta) \sin (2 \theta) & 0 \\
\sin (2 \theta) & \cos (2 \theta) \sin (2 \theta) & \sin ^{2}(2 \theta) & 0 \\
0 & 0 & 0 & 1
\end{array}\right],
$$

From Eq. (2), we can get the equation for determination of required Stokes parameters in the matrix form:

$\mathbf{S}=\mathbf{A}^{-1} \cdot \mathbf{I}$,

where $\mathbf{A}^{-1}$ is the inverse matrix to the characteristic matrix of polarimeter $\mathbf{A}$, which in this case, substituting Eqs. (3) and (4) in Eq. (2), will have the following form:

$$
\mathbf{A}=\left[\begin{array}{cccc}
0.5 & 0.5 & 0 & 0 \\
0.5 & -0.5 & 0 & 0 \\
0.5 & 0 & 0.5 & 0 \\
0.5 & 0 & -0.5 & 0 \\
0.5 & 0 & 0 & 0.5 \\
0.5 & 0 & 0 & -0.5
\end{array}\right] .
$$

We assume that positioning of polarizer and phase plate occur with errors $\Delta \theta$ and $\Delta \alpha$, correspondingly. Besides, we assume also the deviations of phase shift from $\delta=\lambda / 4$ with value $\Delta \delta$, and errors of intensity measurement $\Delta I$ (additive noise) take place.

Taking into account these values of errors $\Delta \theta, \Delta \alpha$, $\Delta \delta$ and $\Delta I$ to estimate the individual measurement errors of each of four Stokes parameters, we use the following relation [25]:

$$
\Delta F_{i}\left(y_{1}, y_{2}, \ldots, y_{N}\right)=\sqrt{\sum_{n=1}^{N}\left(\frac{\partial F_{i}\left(y_{n}^{0}\right)}{\partial y_{n}} \Delta y_{n}\right)^{2}},
$$

where $F_{i}\left(y_{1}, y_{2}, \ldots, y_{N}\right)$ denote the parameters calculated basing on the measured parameters $y_{n} \cdot y_{n}^{0}$ is an exact value of measured parameter $y_{n}$.

Accordingly to Eqs. (5) and (7), the values of errors for Stokes parameters can be written in the following form:

$$
\Delta S_{i}=\sqrt{\sum_{k=1}^{6}\left(\frac{\partial S_{i}}{\partial \theta_{k}} \Delta \theta\right)^{2}+\left(\frac{\partial S_{i}}{\partial \delta} \Delta \delta\right)^{2}+\left(\frac{\partial S_{i}}{\partial \alpha} \Delta \alpha\right)^{2}+\left(\frac{\partial S_{i}}{\partial I} \Delta I\right)^{2}} .
$$

Using Eq. (8) and setting the values of errors as $\Delta \delta=0.5^{\circ}, \Delta \alpha=0.2^{\circ}, \Delta \theta=0.2^{\circ}$, and $\Delta I=I \cdot 0.001$ (here $S_{1}=I=1$ is the light intensity of the beam incident on the phase plate (see Fig. 1)), we derive the dependences of the measurement errors for Stokes parameters on the incident light polarization state ( $\beta$ is an azimuth and $\varepsilon$ is an ellipticity angle for polarization ellipse of input light) (see Fig. 2).

$$
\mathbf{M}_{P h P l}(\delta, \alpha)=\left[\begin{array}{cccc}
1 & 0 & 0 & 0 \\
0 & \cos ^{2}(2 \alpha)+\sin ^{2}(2 \alpha) \cos (\delta) & \cos (2 \alpha) \sin (2 \alpha)((1-\cos (\delta))) & -\sin (2 \alpha) \sin (\delta) \\
0 & \cos (2 \alpha) \sin (2 \alpha)(1-\cos (\delta)) & \sin ^{2}(2 \alpha)+\cos ^{2}(2 \alpha) \cos (\delta) & \cos (2 \alpha) \sin (\delta) \\
0 & \sin (2 \alpha) \sin (\delta) & -\cos (2 \alpha) \sin (\delta) & 0
\end{array}\right]
$$




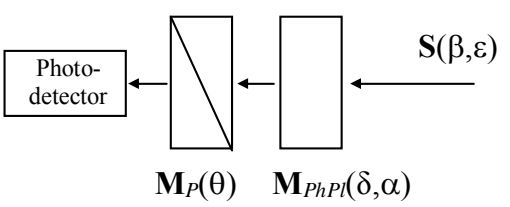

Fig. 1. Stokes-polarimeter setup.

It can be seen that errors in parameters strongly depend on the polarization state of incident light. Mean errors (shown at the bottom of Fig. 2 as the vector $\overline{\Delta \mathbf{S}}$ ) are equal for parameters $S_{1}, S_{2}$ and $S_{3}$, and grow for parameters $S_{4}$ by 2.6 times.

The range of error changes (shown as the vector $\Delta \mathbf{S}_{p p}$ at the bottom of Fig. 2) is equal for parameters $S_{2}$ and $S_{3}$. For $S_{1}$ range of error changes is two times less than that for the parameters $S_{2}$ and $S_{3}$, but for $S_{4}$ is approximately 1.6 times larger. The value of the measurement error for Stokes parameters increases upon the average with decrease of the ellipticity angle $\varepsilon$ of incident light.

The Stokes parameters are determined with minimum errors for light with polarization closing to the circular polarization $(\varepsilon= \pm \pi / 4)$. Also it can be seen that the errors for $S_{1}$ and $S_{3}$ for input polarization with the azimuth $\beta= \pm 45^{\circ}$ relatively to analyzer $\left(\theta=0^{\circ}\right)$ become minimum, and it is practically irrespective to the ellipticity angle. A similar situation with the parameter $S_{2}$ is observed for polarization of input light with the azimuth $\beta=0 \pm 90^{\circ}$.

\section{Stokes polarimeter based on the method of four intensities}

On the assumption of dimension of the Stokes vector, the set of equations for determining the vector parameters should contain four equations. This set of equations can be obtained by measuring the light intensity $I_{i}$ after the polarizer $\mathbf{M}_{P}\left(0^{\circ}\right)$ (Fig. 1) with the fixed orientation $\theta=0^{\circ}$ and phase plate $\mathbf{M}_{P h P l}\left(\delta, \alpha_{i}\right)$ with the fixed phase shift $\delta$ and four angular positions $\alpha_{i}$. The expression for a light intensity $I_{i}$ incident on the detector can be written in the form:

$$
\begin{aligned}
& I_{i}\left(\delta, \alpha_{i}, \mathbf{S}\right)=\left[\mathbf{M}_{P}(0) \mathbf{M}_{P h P l}\left(\delta, \alpha_{i}\right) \mathbf{S}\right]_{0}= \\
& =S_{1}+\left(\cos \left(2 \alpha_{i}\right)^{2}+\sin \left(2 \alpha_{i}\right)^{2} \cos (\delta)\right) S_{2}+ \\
& +\cos \left(2 \alpha_{i}\right) \sin \left(2 \alpha_{i}\right)(1-\cos (\delta)) S_{3}-\sin \left(2 \alpha_{i}\right) \sin (\delta) S_{4} .
\end{aligned}
$$

Thus, from Eq. (9) for Stokes parameters $S_{i}$ we get the following matrix equation:
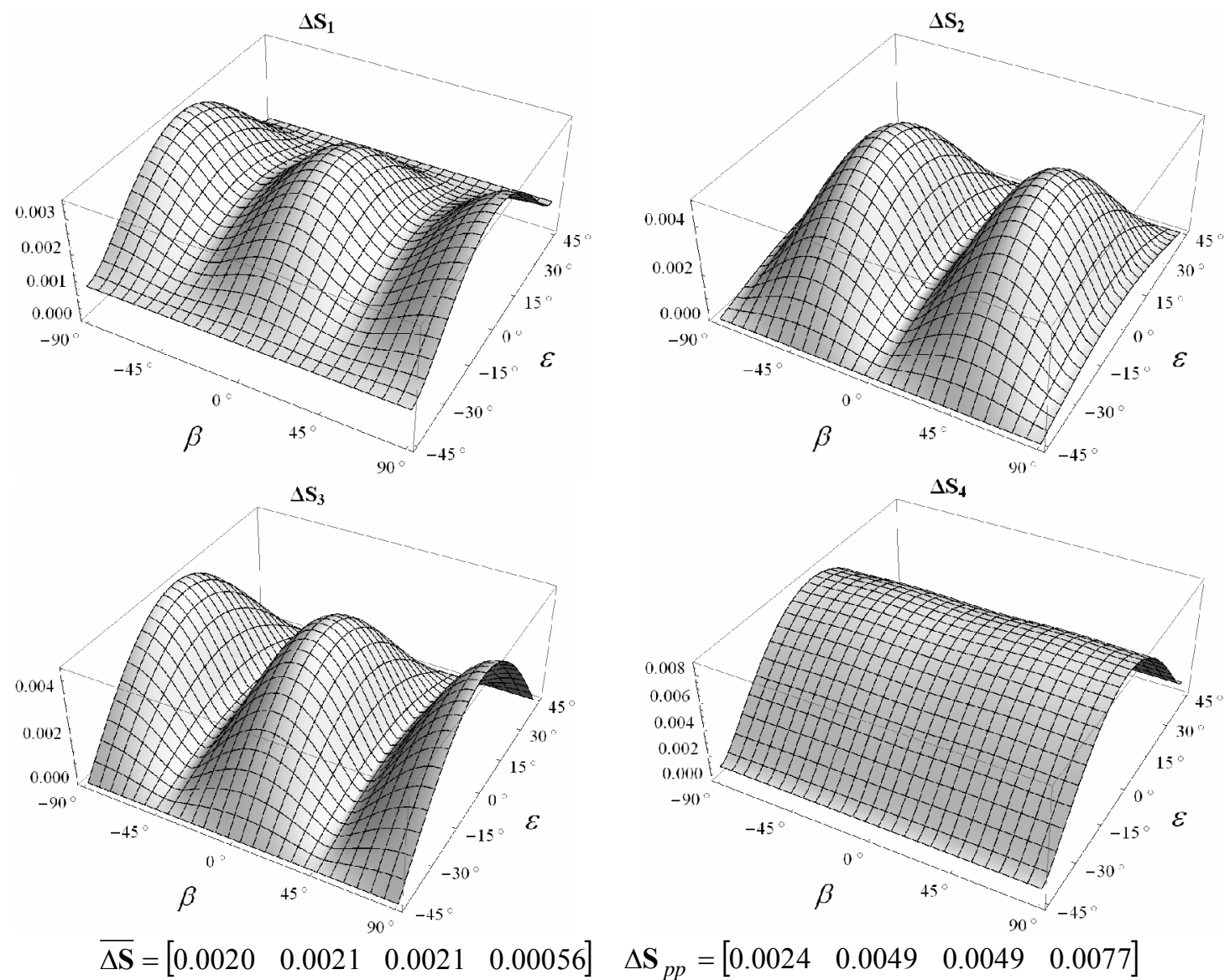

Fig. 2. Dependences of the values of $\Delta \mathrm{S}_{\mathrm{i}}$ on polarization of input light for the Stokes polarimeter based on phenomenological definition of Stokes parameters. 


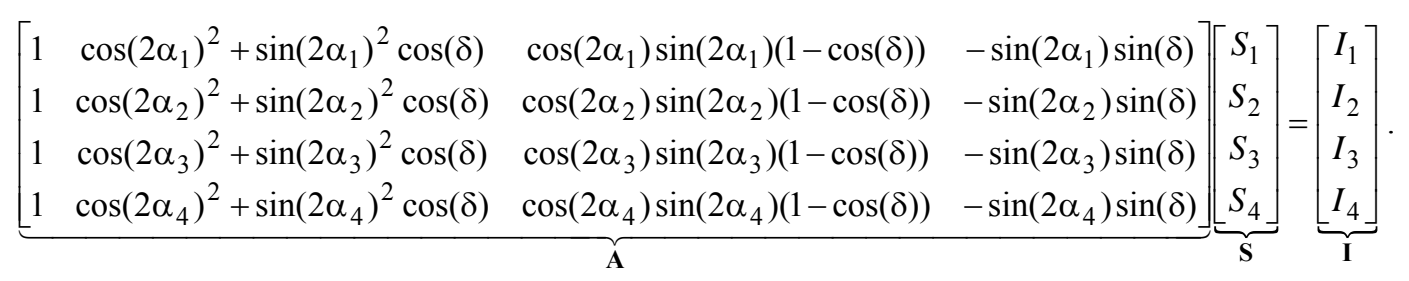

It follows from Eq.(10) that the measurement errors $\Delta S_{i}$ are determined by errors $\Delta I_{i}, \Delta \delta$ and $\Delta \alpha_{i}$. Values of the Stokes parameters can be derived as functions of $I_{i}$, $\delta, \alpha_{i}$ from Eq. (10) making use of Eq. (5).

Then, in conformity with Eq. (7), $\Delta S_{i}$ are:

$$
\Delta S_{i}=\sqrt{\left(\frac{\partial S_{i}}{\partial \delta} \Delta \delta\right)^{2}+\sum_{k=1}^{4}\left[\left(\frac{\partial S_{i}}{\partial \alpha_{k}} \Delta \alpha\right)^{2}+\left(\frac{\partial S_{i}}{\partial I_{k}} \Delta I\right)^{2}\right]} .
$$

As it was in the previous case, $\Delta S_{i}$ depend on the polarization state $(\beta, \varepsilon)$ of input light. Fig. 3 presents the dependences of $\Delta S_{i}$ as functions of $(\beta, \varepsilon)$, which gives the following values for the parameters of the phase plate: $\delta=132^{\circ}, \alpha_{i}=\left(-51.7^{\circ},-15^{\circ}, 15^{\circ}, 51.7^{\circ}\right)$ that were defined in [16], and imperfections of polarimeter parameters: $\Delta \delta=0.5^{\circ}, \Delta \alpha=0.2^{\circ}, \Delta I=I_{0} \cdot 0.001$.

It can be seen that locations of maximum and minimum for errors of Stokes parameters $\Delta S_{i}$ are antisymmetric with respect to $\beta=0^{\circ}$.

As it follows from values for the vector $\overline{\Delta \mathbf{S}}$ (see Fig. 3), the Stokes parameter $S_{4}$ was measured in this case with the largest errors. The parameter $S_{2}$ is characterized by the greatest range $\Delta \mathbf{S}_{p p}$ depending on the polarization state of input light. We show that a uniform distribution of angular orientations of phase plate $\alpha_{i}$ (say, for example, $\alpha_{i}=\left(-60^{\circ},-30^{\circ}, 30^{\circ}, 60^{\circ}\right)$ ) removes the asymmetric locations of maximum and minimum values of $\Delta S_{i}$, but the mean error $\overline{\Delta \mathbf{S}}$ is increased essentially.

\section{Dynamic Stokes polarimeter}

Dynamic Stokes-polarimeter contains a retarder $\mathbf{M}_{P h P l}(\delta, \omega t)$ before the polarizer P (see Fig. 1) rotating with a fixed frequency $\omega$. Thus, in this case the detector signal has the form:

$$
\begin{aligned}
& I(\delta, \omega t, \mathbf{S})=\left[\mathbf{M}_{P}(0) \mathbf{M}_{P h P l}(\delta, \omega t) \mathbf{S}\right]_{1}= \\
& =S_{1}+S_{2}\left(\cos (2 \omega t)^{2}+\cos (\delta) \sin (2 \omega t)^{2}\right)+ \\
& +0.5 S_{3}(1-\cos (\delta)) \sin (4 \omega t)-S_{4} \sin (\delta) \sin (2 \omega t) .
\end{aligned}
$$

Eq. (12) reduces to the following set of equations for Stokes parameters $S_{i}$ :

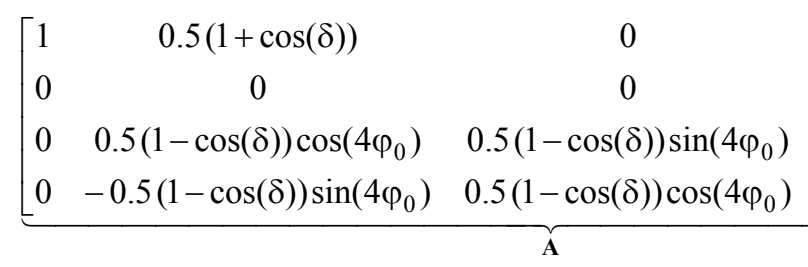

Here, $\varphi_{0}$ is the initial position of the phase plate; $a_{k}$, $b_{k}$ are the amplitudes of harmonics $\sin (k \omega t)$ and $\cos (k \omega t)$, respectively:

$$
\begin{aligned}
& a_{0}=\frac{1}{N} \sum_{i=1}^{N} I\left(\delta, \alpha_{i}, \mathbf{S}\right), \\
& a_{k}=\frac{2}{N} \sum_{i=1}^{N} I\left(\delta, \alpha_{i}, \mathbf{S}\right) \cos \left(\frac{2 \pi k}{N}(i-1)\right), \\
& b_{k}=\frac{2}{N} \sum_{i=1}^{N} I\left(\delta, \alpha_{i}, \mathbf{S}\right) \sin \left(\frac{2 \pi k}{N}(i-1)\right),
\end{aligned}
$$

where $N$ is a number of samplings within the period $T=2 \pi / \omega ; \alpha_{i}-$ position of the phase plate at $i$-th sampling.

The analysis shows that minimum values of errors $\Delta \delta$ and $\Delta \varphi_{0}$ are achieved for $\delta=129.6^{\circ}$ and $\varphi_{0}=0^{\circ}$.

It follows from Eqs. (13) and (14) that errors $\Delta S_{i}$ in dynamic Stokes-polarimeter depend on values $\Delta \delta, \Delta \alpha_{i}$ and $\Delta \varphi_{0}$. Thus, in according with Eqs. (13), (14) and (7) the expression for $\Delta S_{i}$ takes the form:

$$
\Delta S_{i}=\sqrt{\left(\frac{\partial S_{i}}{\partial \delta} \Delta \delta\right)^{2}+\left(\frac{\partial S_{i}}{\partial \varphi_{0}} \Delta \varphi_{0}\right)^{2}+\sum_{k=1}^{N}\left[\left(\frac{\partial S_{i}}{\partial \alpha_{k}} \Delta \alpha\right)^{2}+\left(\frac{\partial S_{i}}{\partial I_{k}} \Delta\right)^{2}\right]}
$$

Fig. 4 shows the dependences $\Delta S_{i}$ on polarizations ( $\beta$ and $\varepsilon$ ) of input light, which gives the optimal set of parameters for the phase plate $\mathbf{M}_{P h P l}$ (Fig. 1): $\delta=129.6^{\circ}$ and $\varphi_{0}=0^{\circ}$. The number of samplings was chosen as $N=360$. The error in initial orientation of the phase plate is $\Delta \varphi_{0}=0.2^{\circ}$. Other three values $\Delta I_{i}, \Delta \delta, \Delta \alpha_{i}$ are the same as in previous cases.

As it can be seen from Fig. 4 the parameters $S_{2}$ and

\begin{tabular}{|c|c|c|}
\hline 0 & $\left.S_{1}\right]$ & $\left\lceil a_{0}\right.$ \\
\hline$-\cos \left(2 \varphi_{0}\right) \sin (\delta)$ & $S_{2}$ & $b_{2}$ \\
\hline 0 & $S_{3}$ & $a_{4}$ \\
\hline 0 & $S_{4}$ & $b_{4}$ \\
\hline
\end{tabular}
$S_{3}$ have been determined upon the average with equal errors and are only different in locations of maxima. The mean value of $\overline{\Delta S_{4}}$ is 1.3 times less than that of $\overline{\Delta S_{2}}$ and $\overline{\Delta S_{3}}$. The value of the parameter $S_{1}$ has been determined more precisely as compared with $S_{2}$ and $S_{3}$ (approximately by 2.6 times) and $S_{4}$ (approximately by 1.9 times). The value of errors $\Delta \mathbf{S}_{p p}$ for parameters $S_{2}$ and $S_{3}$ are equal and less than that of the parameter $S_{1}$ by 2.3 times and that of $S_{4}$ by 5.2 times. 


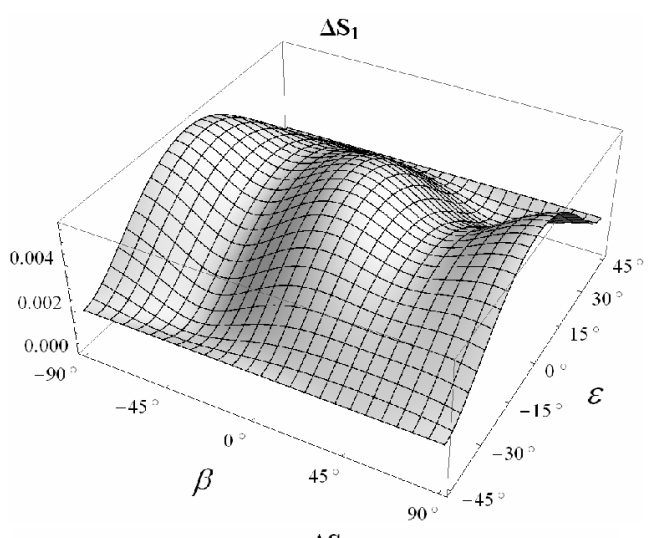

$\Delta \mathbf{S}_{3}$

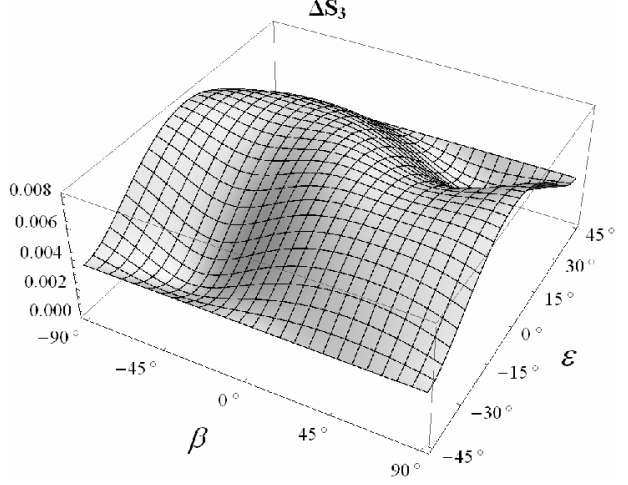

$\overline{\Delta \mathbf{S}}=\left[\begin{array}{llll}0.0036 & 0.0055 & 0.0057 & 0.0076\end{array}\right]$

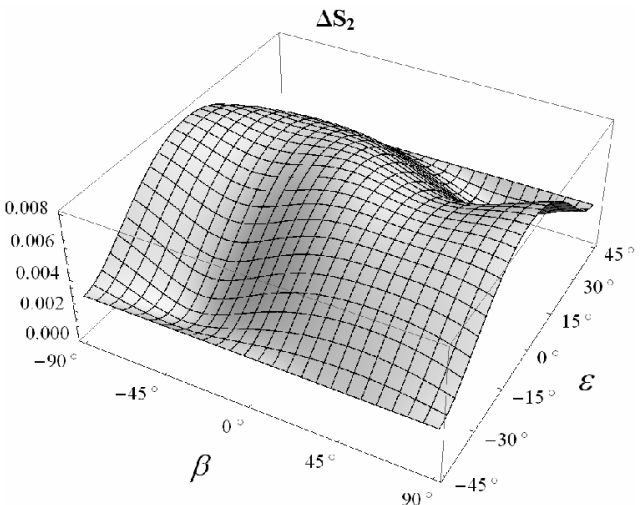

$\Delta \mathbf{S}_{4}$

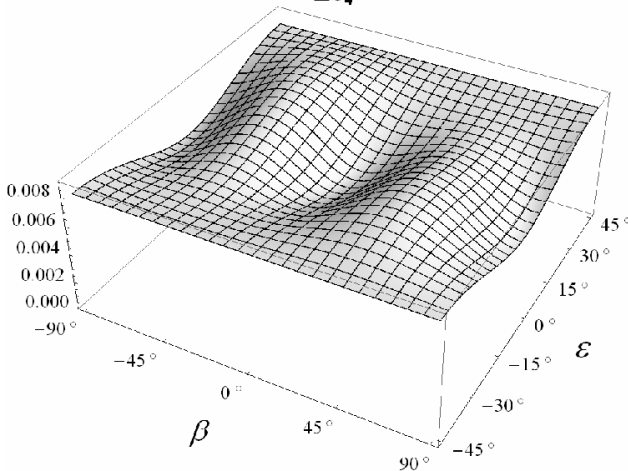

$\Delta \mathbf{S}_{p p}=\left[\begin{array}{llll}0.0042 & 0.0061 & 0.056 & 0.0032\end{array}\right]$

Fig. 3. Dependences of the $\Delta S_{i}$ values on polarization of input light for the Stokes polarimeter based on the method of four intensities.

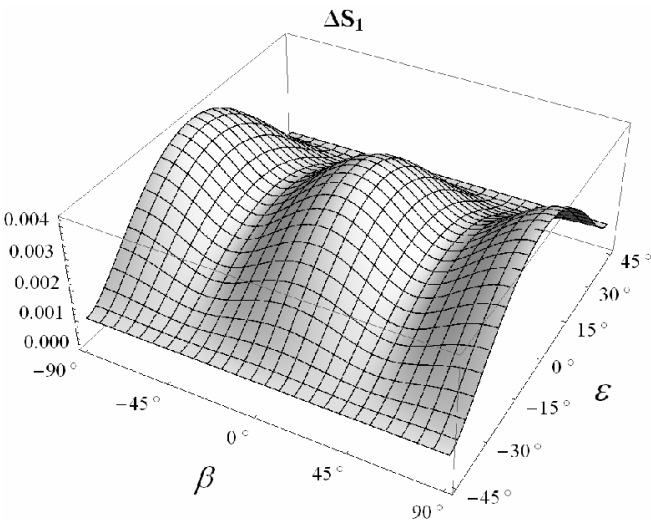

$\Delta \mathbf{S}_{3}$

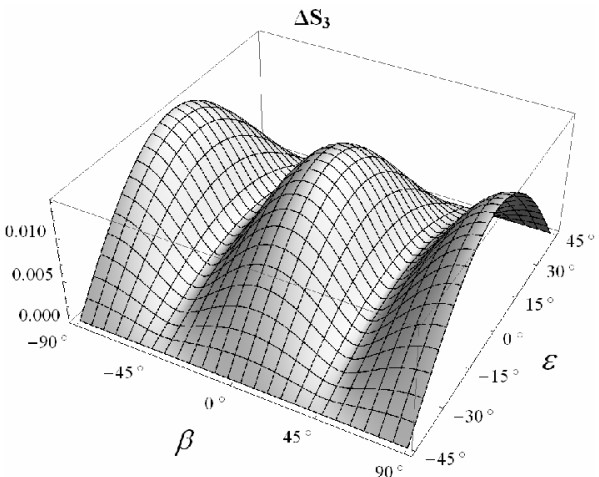

$\overline{\Delta \mathbf{S}}=\left[\begin{array}{llll}0.0024 & 0.0062 & 0.0062 & 0.0046\end{array}\right]$

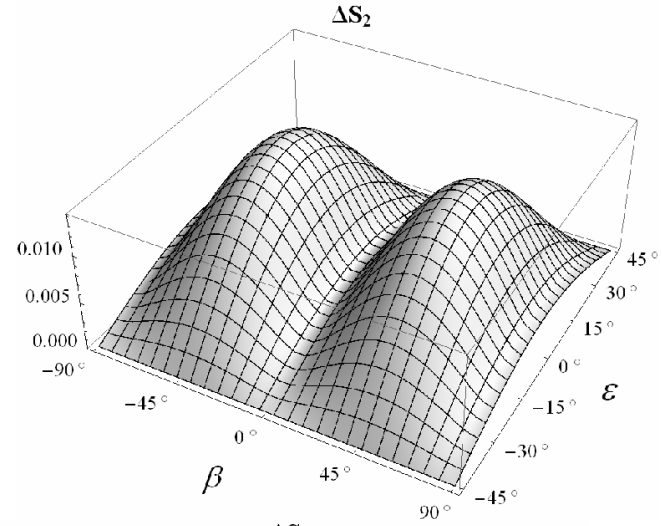

$\Delta \mathbf{S}_{4}$

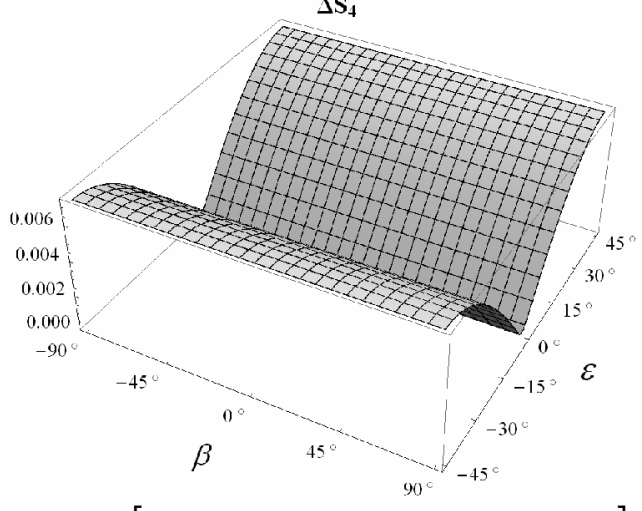

$\Delta \mathbf{S}_{p p}=\left[\begin{array}{llll}0.0032 & 0.0014 & 0.0014 & 0.0073\end{array}\right]$

Fig. 4. Dependences of the $\Delta S_{i}$ values on polarization of input light for the dynamic Stokes polarimeter. 


\section{The effect of measurement errors $\Delta S_{i}$ on determination of polarization parameters of input light}

The given Stokes parameters, $S_{i}$, polarization parameters, intensity $I$, degree of polarization $P$, ellipticity angle $\varepsilon$ and azimuth of polarization ellipse $\beta$ can be determined in the following manner:

$$
\begin{aligned}
& I=S_{1}, \quad P=\frac{\sqrt{S_{2}^{2}+S_{3}^{2}+S_{4}^{2}}}{S_{1}^{2}}, \\
& \varepsilon=\frac{1}{2} \arcsin \left(\frac{S_{4}}{\sqrt{S_{2}^{2}+S_{3}^{2}+S_{4}^{2}}}\right), \quad \beta=\frac{1}{2} \operatorname{arctg}\left(\frac{S_{3}}{S_{2}}\right) .
\end{aligned}
$$

Errors in values of Stokes parameters will be transferred into errors of polarization parameters Eq. (16). To estimate this effect, we use Eq. (7):

$$
\begin{gathered}
\Delta P=\sqrt{\sum_{i=1}^{4}\left(\frac{\partial P}{\partial S_{i}} \Delta S_{i}\right)^{2}}, \quad \Delta \varepsilon=\sqrt{\sum_{i=1}^{4}\left(\frac{\partial \varepsilon}{\partial S_{i}} \Delta S_{i}\right)^{2}}, \\
\Delta \beta=\sqrt{\sum_{i=1}^{4}\left(\frac{\partial \beta}{\partial S_{i}} \Delta S_{i}\right)^{2}} .
\end{gathered}
$$

Since the values $\Delta S_{i}(\beta, \varepsilon)$ are different for different Stokes-polarimeters discussed above, thus, the errors of polarization parameters Eq. (17) are different for different Stokes-polarimeters as well. Fig. 5 exemplifies
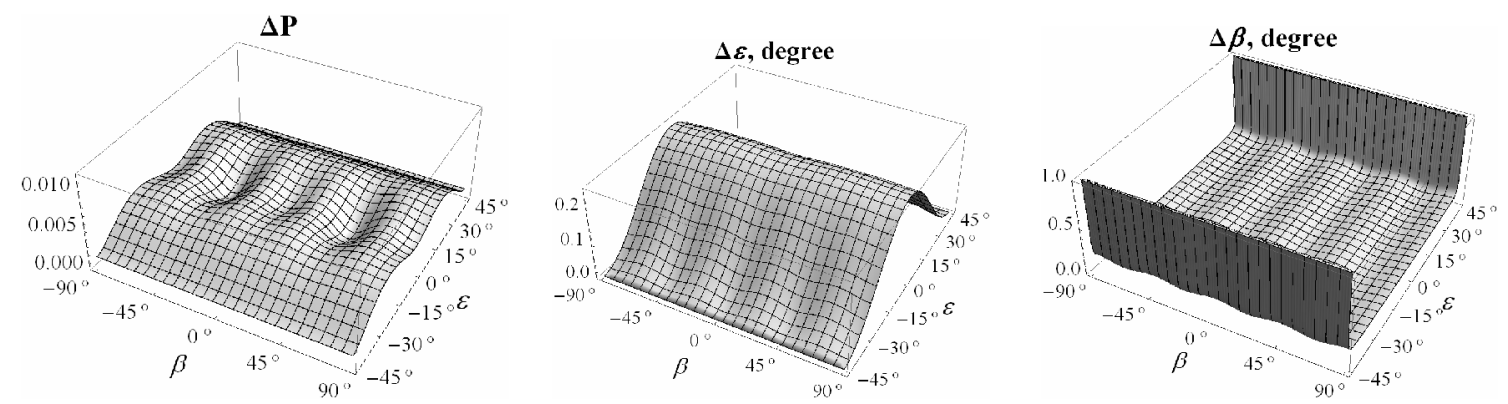

$$
\overline{\Delta P}=0.0040, \Delta P_{p p}=0.0039 ; \overline{\Delta \varepsilon}=0.13^{\circ}, \Delta \varepsilon_{p p}=0.25^{\circ}, \overline{\Delta \beta}=0.13^{\circ}, \Delta \beta_{p p}=0.10^{\circ} \text {. }
$$

a)
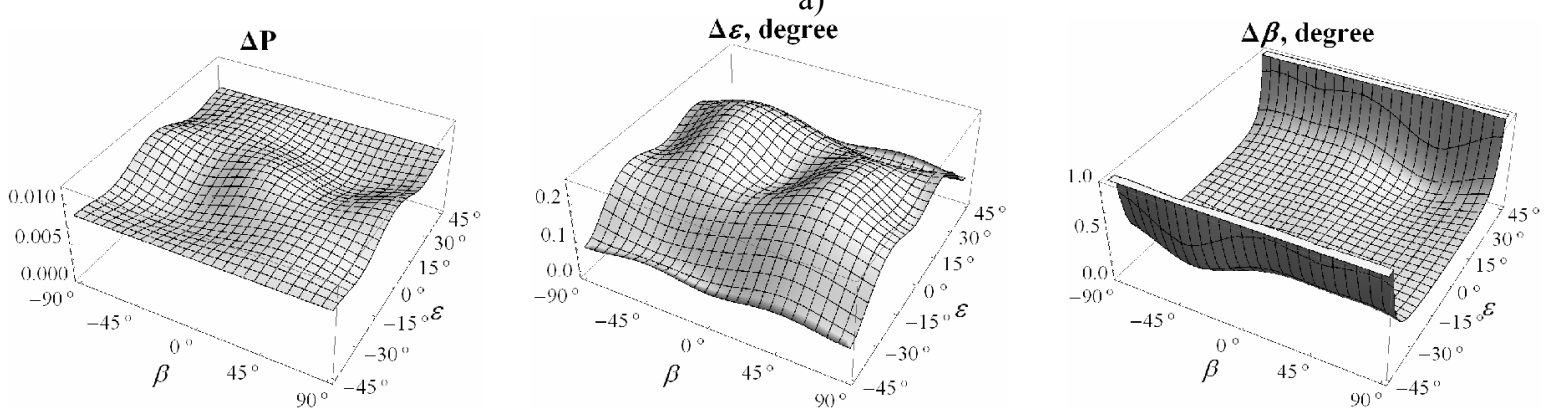

$\overline{\Delta P}=0.0083, \Delta P_{p p}=0.0034, \overline{\Delta \varepsilon}=0.17^{\circ}, \Delta \varepsilon_{p p}=0.16^{\circ}, \overline{\Delta \beta}=0.26^{\circ}, \Delta \beta_{p p}=0.15^{\circ}$

b)
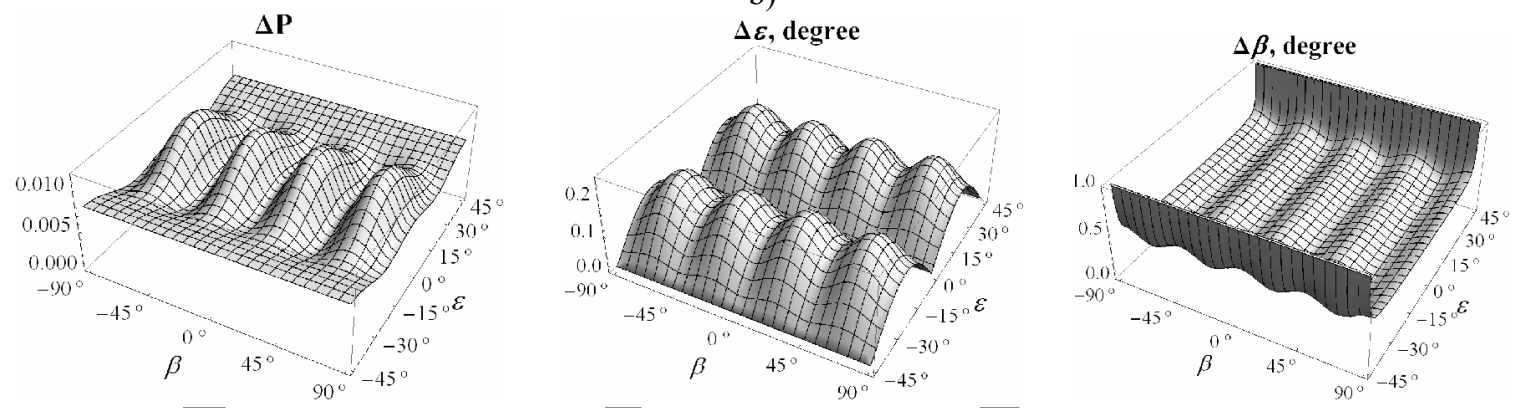

$$
\overline{\Delta P}=0.0076, \Delta P_{p p}=0.0058, \overline{\Delta \varepsilon}=0.11^{\circ}, \Delta \varepsilon_{p p}=0.18^{\circ}, \overline{\Delta \beta}=0.37^{\circ}, \Delta \beta_{p p}=0.31^{\circ}
$$

c)

Fig. 5. Dependences of the $\Delta P, \Delta \varepsilon, \Delta \beta$ values for different polarizations of input light: (a) Stokes polarimeter based on phenomenological definition of Stokes parameters; (b) dynamic Stokes polarimeter; (c) Stokes polarimeter based on the method of four intensities. 
the dependences $\Delta P(\beta, \varepsilon), \Delta \varepsilon(\beta, \varepsilon)$ and $\Delta \beta(\beta, \varepsilon)$ for three types of polarimeters discussed above.

It follows from Fig. 5 that the ellipticity of polarization ellipse has been determined with the minimum errors by dynamic Stokes-polarimeter. Whereas, the polarization degree $P$ and azimuth of polarization ellipse $\beta$ are determined with minimum errors by Stokes polarimeter based on phenomenological definition of Stokes parameters. For Stokes polarimeter based on the method of four intensities, the effect of polarization of input light on the errors $\Delta P, \Delta \varepsilon$ and $\Delta \beta$ is minor. More sensitive to the polarization state of input light is the dynamic Stokes-polarimeter.

\section{Conclusion}

In summary, we have analyzed the errors in determination of the Stokes parameters for three types of Stokes polarimeters: (i) Stokes polarimeter based on phenomenological definition of Stokes parameters; (ii) Stokes polarimeter based on the method of four intensities; (iii) dynamic Stokes polarimeter. Also, we have analyzed the transfer of the errors for Stokes parameters $\Delta S_{i}$ into the errors in determination of polarization parameters (degree of polarization $P$, ellipticity angle $\varepsilon$ and azimuth of polarization ellipse $\beta$ ) of input light. We have shown that in general case the Stokes parameters are determined with a different accuracy both in scope of one measurement strategy and by different measurement strategies. The Stokes vector is measured as a whole with minimum errors by Stokes polarimeter based on phenomenological definition of Stokes parameters: $\sum_{i=1}^{4} \overline{\Delta S}_{i} / 4=0.0017$. The dynamic Stokes polarimeter and Stokes polarimeter based on the method of four intensities measure the Stokes vector with a somewhat higher value of errors: $\sum_{i=1}^{4} \overline{\Delta S}_{i} / 4=0.0049 \quad$ and $\quad \sum_{i=1}^{4} \overline{\Delta S}_{i} / 4=0.0056$, respectively.

This is quite important fact because it means that, under rest equal circumstances, the parameters of polarization of input light are measured with different errors by different polarimeters. Moreover, the errors depend on the polarization state of input light as well. The results obtained for errors of the Stokes parameters allowed to determine that the ellipticity angle $\varepsilon$ is measured more precisely by dynamic Stokespolarimeter, whereas, the azimuth $\beta$ and degree of polarization $P$ are measured more precisely by Stokes polarimeter based on phenomenological definition of Stokes parameters.

The results derived in this paper can be useful for choosing the measurement strategy for given polarization of input light. Indeed, the Stokes polarimeter based on phenomenological definition of
Stokes parameters is complicated for automatization. The dynamic Stokes polarimeter is evidently unacceptable for imaging Stokes vector measurements [10]. At the same time, the Stokes polarimeter based on the method of four intensities, although characterized by a relatively higher value of errors when determining Stokes parameters, is simple for automatization and promising for imaging Stokes polarimetry.

It is noteworthy that on the assumption of equality of $\Delta S_{i}$ there exist no reasons to prefer one Stokes polarimeter to another. An exception can be very likely made only when concerning to assembling and operating conveniences. Since the accuracy in determination of an individual Stokes parameter is different for different types of polarimeters and, therewith, it depends on polarization of input light, then all these strongly motivates the choice of polarimeter type to provide minimum errors in determination of polarization parameters (ellipticity angle $\varepsilon$, azimuth $\beta$, and degree of polarization $P$ ). This gains even more important significance, when the choice of measurement strategy can be made only by software [27].

\section{References}

1. Ch. Brosseau, Fundamentals of Polarized Light. A Statistical Optics Approach. North-Holland Publishing Company, New York, p. 406, 1998.

2. M.I. Mishchenko, L.D. Travis, A.A. Lacis, Scattering, Absorption, and Emission of Light by Small Particles. Cambridge University Press, Cambridge, p. 449, 2002.

3. S.L. Durden, J.J. van Zyl, and H.A. Zebker, The unpolarized component in polarimetric radar observations of forested areas // IEEE Trans. Geoscience and Remote Sensing 28, p. 268-271 (1990).

4. S.L. Jacques, R.J. Roman and K. Lee, Imaging skin pathology with polarized light // J. Biomed. Opt. 7, p. 329-340 (2002).

5. S.P. Morgan, I.M. Stockford, Surface-reflection elimination in polarization imaging of superficial tissue // Opt. Lett. 28, p. 114-116 (2003).

6. J.M. Schmitt, A.H. Gandjbakhche and R.F. Bonner, Use of polarized light to discriminate short path photons in a multiply scattering medium // Appl. Opt. 31, p. 6535-6546 (1992).

7. P.S. Hauge, Resent developments in instrumentation in ellipsometry // Surf. Sci. 96, p. $108-140$ (1980).

8. A.M. Shutov, The optical systems of devices for measuring the parameters of polarized radiation // Optiko-mekhanicheskaya promyshlennost' $\mathbf{1 1}$ p. 52-56 (1985) (in Russian).

9. R.M.A. Azzam, Mueller-matrix ellipsometry: review // Proc. SPIE 3121, p. 396-405 (1997).

10. J.S. Tyo, D.L. Goldstein, D.B. Chenault and J.A. Shaw, Review of passive imaging polarimetry 
for remote sensing applications // Appl. Opt. 45(22), p. 5453-5469 (2006).

11. R.M.A. Azzam, Arrangement of four photodetectors for measuring the state of polarization of light // Opt. Lett. 10, p. 309-311 (1985).

12. M. Born and E. Wolf, Principles of Optics. Pergamon, New York, p. 554, 1975.

13. A. Ambirajan and D.C. Look, Optimum angles for a polarimeter: part $1 / /$ Opt. Eng. 34, p. 1651-1655 (1995).

14. P.S. Hauge and F.H. Dill, A rotating-compensator Fourier ellipsometer // Opt. Communs. 14, p. 431437 (1975).

15. P.S. Huge, Generalized rotating-compensator ellipsometry // Surf. Sci. 56, p. 148-160 (1976).

16. D.S. Sabatke, M.R. Descour and E.L. Dereniak, Optimization of retardance for a complete Stokes polarimeter // Opt. Lett. 25(11), p. 802-804 (2000).

17. I.J. Vaughn and B.G. Hoover, Noise reduction in a laser polarimeter based on discrete waveplate rotations // Opt. Exp. 16(3), p. 2091-2108 (2006).

18. R.M.A. Azzam, I.M. Elminyawi and A.M. El-Saba, General analysis and optimization of the fourdetector photopolarimeter // J. Opt. Soc. Amer. A 5, p. 681-689 (1988).

19. V.A. Dlugunovich, V.N. Snopko and O.V. Tsaryuk, Analysis of a method for measuring polarization characteristics with a Stokes polarimeter having a rotating phase plate // J. Opt. Technol. 68(4), p. 269-273 (2001).

20. J.S. Tyo, Noise equalization in Stokes parameter images obtained by use of variable-retardance polarimeters // Opt. Lett. 25, p. 1198-1200 (2000).

21. S.N. Savenkov, Optimization and structuring of the instrument matrix for polarimetric measurements // Opt. Eng. 41, p. $965-972$ (2002).

22. V.A. Dlugunovich, E.A. Kuplevich and V.N. Snopko, Minimizing the measurement error of the Stokes parameters when the setting angles of a phase plate are varied // J. Opt. Technol. 67(9), p. $792-800$ (2000).

23. J.S. Tyo, Design of optimal polarimeters: maximization of signal-to-noise ratio and minimization of systematic errors // Appl. Opt. 41, p. 619-630 (2002).

24. J. Zallat, S. Ainouz, and M.Ph. Stoll, Optimal configurations for imaging polarimeters: impact of image noise and systematic errors // J. Opt. A 8, p. 807-814 (2006).

25. J.R. Taylor, An Introduction to Error Analysis, 2 ed. Univ. Sci. Books, Mill Valley, p. 349, 1997.

26. F.R. Gantmacher, The Theory of Matrices. AMS Chelsea Publishing, Reprinted by Amer. Mathemat. Soc., p. 660, 2000.

S.N. Savenkov, Yu.A. Skoblya, K.E. Yushtin, and A.S. Klimov, Mueller matrix polarimetry with multi-purpose polarization transducer // Proc. SPIE 5772, p. 77-85 (2005). 